\title{
STREAMING CIRCUMNUCLEAR GAS OF THE SEYFERT 2 GALAXY NGC 5728
}

\author{
Dong-Hoon Son ${ }^{1}$, Siek Hyung ${ }^{2}$, Seong-Jae LeE ${ }^{2}$, and Pierre Ferruit ${ }^{3}$ \\ ${ }^{1}$ Korea Astronomy and Space Science Institute 61-1, Hwaam-dong, Yuseong-gu, Daejeon, Korea 305-348 \\ E-mail:dhson@kasi.re.kr \\ 2 School of Science Education (Astronomy), Chungbuk National Univ., Chungbuk 361-763, Korea \\ E-mail: hyung,seong@chungbuk.ac.kr \\ 3 CRAL - Observatoire de Lyon, 9 avenue Charles Andre, 69561 Saint Genis Laval cedex, France \\ E-mail: pierre.ferruit@obs.univ-lyon1.fr \\ (Received September 18, 2009; Accepted Octorber 12, 2009)
}

\begin{abstract}
We investigated the circumnuclear region of the Seyfert 2 galaxy NGC 5728, using the CFHT $3.6 \mathrm{~m}$ OASIS [S II], [O III] \& H $\beta$, spectral images complemented with the IUE spectra. The physical condition of the circumnuclear zone has been derived: the gas density (indicated by $[\mathrm{S}$ II] $6716 / 31$ ratio) around the $\mathrm{C}$ core is generally similar to that around the NW core, i.e., $\sim 500 \mathrm{~cm}^{-3}$. However, there appears to be evidence of a higher density shell in front of the NW core, $\sim 10^{4} \mathrm{~cm}^{-3}$ at $-250 \mathrm{~km} \mathrm{~s}^{-1}$. The IUE Si III]1892/C III]1909 ratio implies a possible presence of a broad emission region of gas densities of $\sim 10^{10} \mathrm{~cm}^{-3}$. The SE cone and surrounding area show several prominent features, while the NW cone does not show any particular structure: we identified three prominent blobs in the SE cone and one possible candidate in the NW cone. The outflow activities exist within the relatively large conic opening angle. We discussed the possibility of inflow or outflow activities of blobs found in the circumnuclear region of NGC 5728. The gas around two cores, two cones, and several blobs, is likely to be excited by the AGN hot source(s).
\end{abstract}

Key words : individual: NGC 5728 — galaxies: kinematics and dynamics — galaxies: Seyfert: nuclei - galaxies: emission lines

\section{INTRODUCTION}

There have been numerous studies to discover the characteristics of Active Galactic Nuclei (AGN) and the kinematical structure in the circumnuclear zone of NGC 5728, such as Hubble Space Telescope (HST) optical images by Wilson et al. (1993), radio images by Schommer et al. (1988), and spectra by Mediavilla \& Arribas (1995). The HST images show some evidence of unified model geometry, i.e., an extended ionized biconical zone $\sim 1.8 \mathrm{kpc}$ in extent along the minor axis direction of the host galaxy (Wilson et al. 1993). Schommer et al. (1988) noticed the starburst activity present in the $5^{\prime \prime}$ inner ring. The kinematical study by Rubin (1980), showed the central region might expand and rotate. She assumed the center of this gas flow activity to be at the main core, i.e., the $\mathrm{C}$ core. Another 2D spectral study by Arribas \& Mediavilla (1993) pointed out there is a kinematical center at a distance of $1^{\prime \prime}$ north-west of the $\mathrm{C}$ core.

Most recently, Son et al. (2009, hereafter Paper I) investigated the $12^{\prime \prime} \times 10^{\prime \prime}$ nucleus region of NGC 5728 with the 3D, Optically Adaptive System for Imaging Spectroscopy (OASIS) at the CFHT $3.6 \mathrm{~m}$ telescope.
Paper I showed that the 5!" $4 \mathrm{NW}$ inner ring in $\mathrm{H} \alpha$ image and confirmed its expanding and rotating motion centering at the kinematical center between two main cores. Paper I also found a $3^{\prime \prime}$ rotating inflow disk structure whose plane is different from the galactic disk. This $3^{\prime \prime}$ inflow + rotation disk model interpretation contradicts Rubin (1980)'s interpretation. For the same zone, the interpretations on the kinematical structure, i.e., inclination and kinematical center, are very different in the two studies. We reanalyze the OASIS data of NGC 5728 to study the physical condition of the circumnuclear zone and the kinematical behavior of minute structures, i.e., blobs. Our objective is to provide some additional information to Paper I on the circumnuclear region of NGC 5728. Various [O III] radial velocity slice contour images have been carefully analyzed.

In $\S 2$, the density distribution and $[\mathrm{O}$ III $] / \mathrm{H} \beta$ ratio map of the circumnuclear zone are presented. In $\S 3$, we present the various monochromatic images and discuss the inflow or outflow activity of the blobs. In $\S 4$, we give a brief summary of our study.

Corresponding Author: S. Hyung 

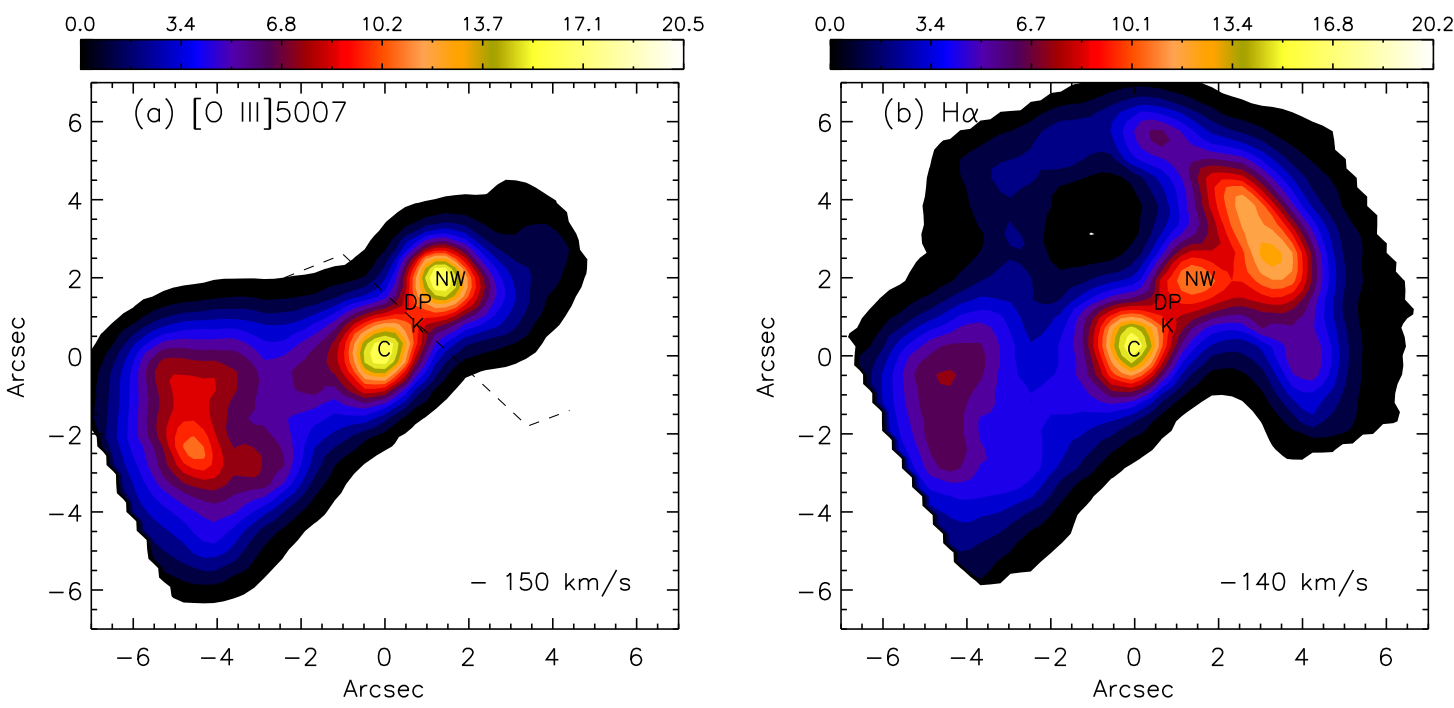

Fig. 1.- $[\mathrm{O}$ III $] 5007$ and $\mathrm{H} \alpha$ images at $\mathrm{V}_{\mathrm{r}}=-150 \&-140 \mathrm{~km} \mathrm{~s}^{-1}$. [O III] $] 5007(5051-5052 \AA: 1 \AA$ or $60 \mathrm{~km} \mathrm{~s}$ bandwidth), $\mathrm{H} \alpha\left(6621-6623 \AA\right.$ : $2 \AA$ or $92 \mathrm{~km} \mathrm{~s}^{-1}$ bandwidth). See text and Paper I for the exact coordinates of C, K, DP, \& NW. Flux unit above color bars: $10^{-19} \mathrm{~W} \mathrm{~m}^{-2} \operatorname{arcsec}^{-2}$.

\section{OASIS AND IUE SPECTRAL DATA}

We observed the Seyfert 2 galaxy NGC $5728 \mathrm{nu}-$ cleus with the 3.6-m CFHT telescopic 3D spectroscope OASIS (2001 March 15). The spatial sampling of each

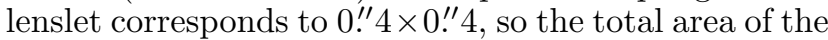
sky covered was about $15^{\prime \prime} \times 12^{\prime \prime}$ with a total of 1100 lenslets of OASIS. The spectral resolution is about 1.9 $\AA$ on each $13.5 \mu \mathrm{m}$ EEV1 CCD pixel. Hence the spectral coverages are $4800-5530 \AA$ in OASIS grism MR1 \& $6220-6990 \AA$ in MR2. The total exposure times are 60 \& 30 minutes in MR1 \& MR2, respectively. See Paper I for detailed explanation of the data reduction.

Fig. 1 presents [O III $] 5007$ and $\mathrm{H} \alpha$ images at nearly the same approaching radial velocity, i.e., $\mathrm{V}_{\mathrm{r}}=-150$ $\&-140 \mathrm{~km} \mathrm{~s}^{-1}$. The radial velocities discussed in this study will be given relative to the system velocity, $\mathrm{V}_{\mathrm{sys}}=c z=2838.14 \mathrm{~km} \mathrm{~s}^{-1}$ (see Paper I). In Fig. 1, we add notations of $\mathrm{C}, \mathrm{NW}, \mathrm{K}, \& \mathrm{DP}$, which indicate the main core, NW core, kinematical center, and one of the double-peaked (DP) line profile zone. The celestial coordinates are given relative to the main core, $\mathrm{C}$ (see Paper I). After correcting the above system velocity, the observed wavelength of $5053.86 \AA$ will become the laboratory wavelength, i.e., [O III] $5006.84 \AA$ or $\mathrm{V}_{\text {sys }}=$ $0 \mathrm{~km} \mathrm{~s}^{-1}$. For simplicity, we adopt a slightly different wavelength $5054 \AA(5053-5055 \AA)$ and $6625 \AA(6624$ - $6626 \AA)$ as the $[\mathrm{O}$ III $] 5007 \& \mathrm{H} \alpha$ references, i.e., $\mathrm{V}_{\mathrm{r}}$ $=0 \mathrm{~km} \mathrm{~s}^{-1}$.

Two cores, C \& NW, appear to be the same in size in the $\mathrm{V}_{\mathrm{r}}=-150 \mathrm{~km} \mathrm{~s}^{-1}$ radial velocity image of [O III $] 5007$ (Fig. 1(a)), but the integrated intensity of the $\mathrm{C}$ core is stronger than the $\mathrm{NW}$ value. In this approaching radial velocity slice, the other prominent emission zone is seen at the south-east zone, which have been identified as a South East (SE) cone in the earlier literatures, e.g., Wilson et al. (1993). The half elliptical circle (or Arc shape) $\mathrm{H} \alpha$ ring is also seen in $\mathrm{H} \alpha$ image of Fig. 1(b). The NW ring is about $\sim 5 !^{\prime \prime} 4$ in radius. The other half circle might be hidden below the relatively thick galactic disk plane. The NW ring was not detected in [O III] or other collision line monochromatic images.

We also add the reverse S-shaped boundary (dashed line) in Fig. 1(a). The DP line profiles result from the extended kinematical structure of two cores. The $6-7^{\prime \prime}$ long, reverse S-shaped boundary, which bisects the blue- and red-shifted line profile dominated spectral zones, runs through the DP zone along the galactic major axis direction (see Paper I).

To show the quality of the spectra, we present spectral scans in Fig. 2 from MR1 \& MR2. Fig. 2(a) \& (b) are extracted from one lenslet at $\mathrm{K}$ position, while Fig. 2(c) \& (d) are from one of the bright ring spots, the $\mathrm{R} 4$ position. Note that the $[\mathrm{O}$ III] lines at the $\mathrm{K}$ position are stronger than that of $\mathrm{H} \beta$ whereas the opposite is true at R4. Paper I concluded that the $\mathrm{K}$ and two core emissions are related to the AGN activity of the two cores, while the ring emission is mainly from the stellar origin, e.g., star burst activity. Various other lines have been registered in our OASIS observations, but we analyze [S II], [O III], \& $\mathrm{H} \beta$ spectral images in this study.

Fig. 2(e) gives the IUE spectrum of NGC 5728, combined from SWP31381 (1987, July 21) and LWP11256 (1987, July 20). Since the IUE spectra were from the 

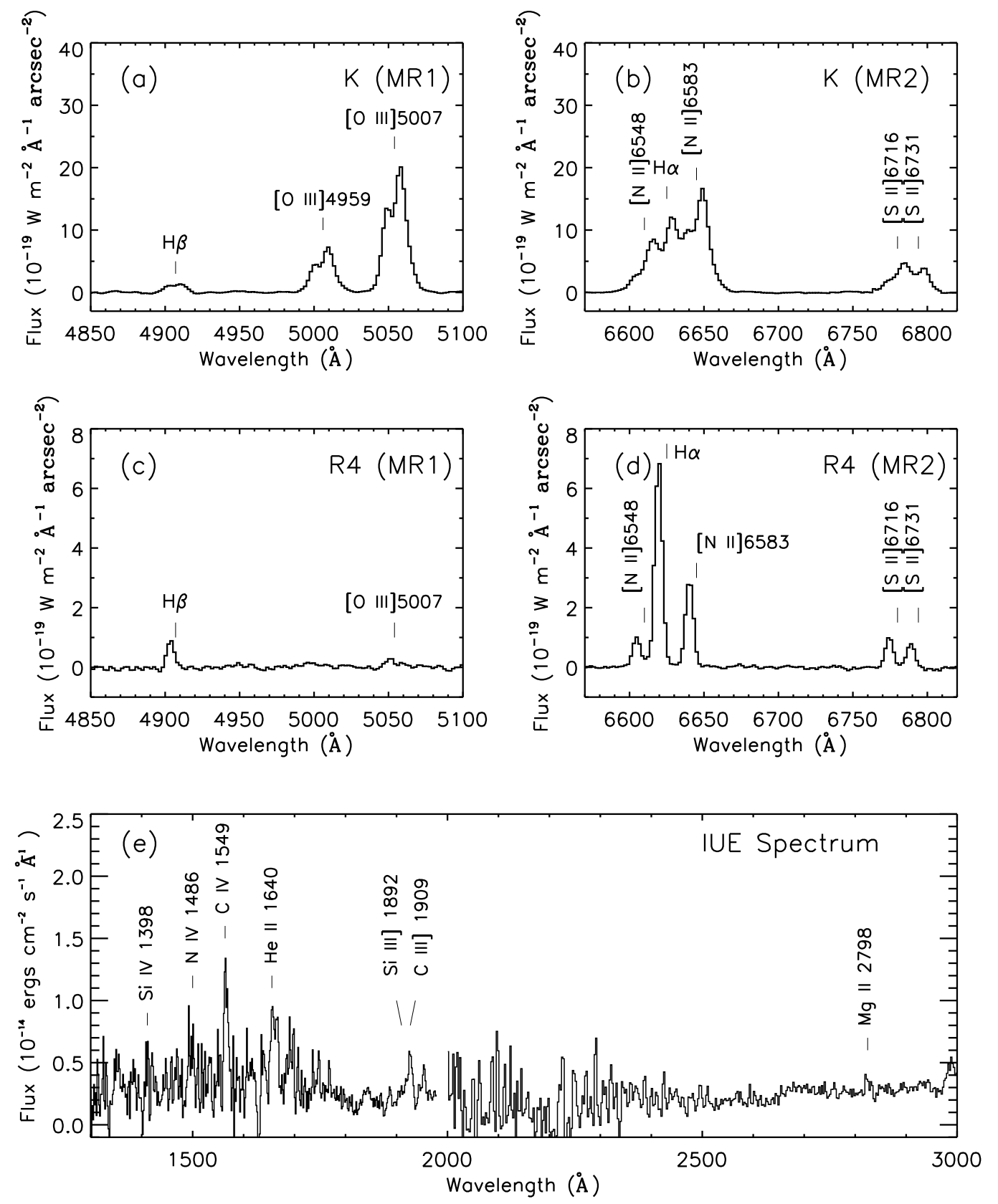

Fig. 2.- OASIS and IUE spectra. (a) - (d) OASIS spectra at K \& R4 positions. (e) IUE spectrum. 

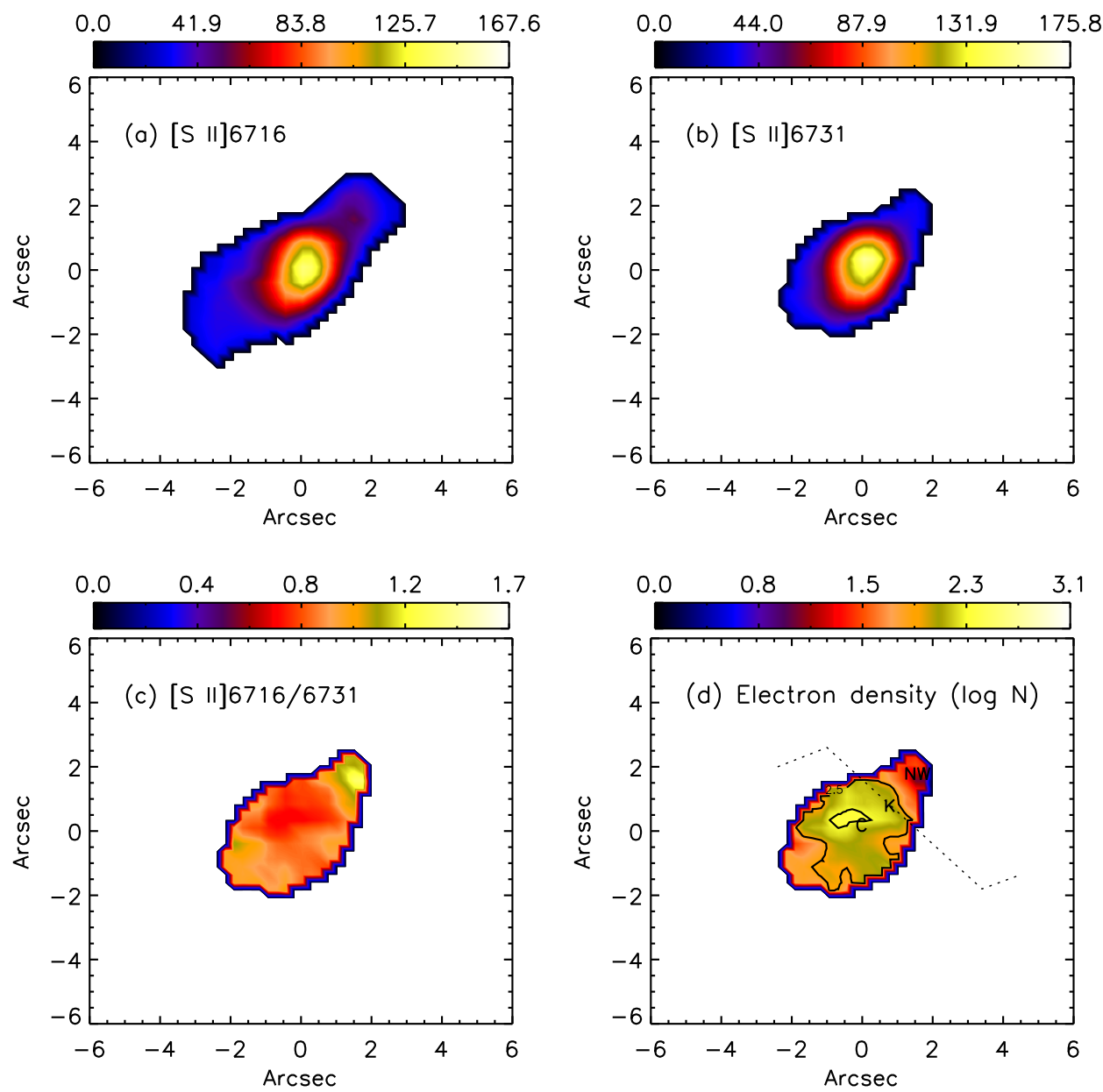

Fig. 3. - $[\mathrm{S} \mathrm{II}]$ ratios and electron density maps. (a) \& (b) $[\mathrm{S} \mathrm{II}]$ within $\mathrm{V}_{\mathrm{r}}=-180-+180 \mathrm{~km} \mathrm{~s}^{-1}$. (c) $[\mathrm{S} \mathrm{II}] 6716 / 6731$ line ratio. (d) logarithmic electron density from (c). C, K, NW, and reverse S-shaped boundary (dotted line) marked.

$10^{\prime \prime} \times 23^{\prime \prime}$ oval area, the spectrum covers a wider area than the optical OASIS spectrum does. Pécontal et al. (1990) reported the possible existence of the Broad Line Region (BLR) component in the $\mathrm{H} \alpha$ secured with TIGER (the prototype 3D spectrometry) spectral data. However, Paper I showed that the BLR-like $\mathrm{H} \alpha$ components are actually the combined line profiles involving two main cores. These two main cores might have independent super-massive black holes. Although the optical region of the OASIS spectral data did not confirm the presence of BLR in Paper I, we are able to get some evidence from the IUE C III]1909/Si III]1892 line ratio (see Feibelman \& Aller 1987; Hyung et al. 2000). Using the Starlink/DIPSO package, we measure the C III]1909/Si III]1892 line ratio and derive a relatively high electron density, $N_{\epsilon} \sim 1.6 \times 10^{10} \mathrm{~cm}^{-3}$, as expected in other typical Seyfert 1 galaxies. There appears to be an unresolvable BLR core in the circumnuclear region of NGC 5728.

The electron temperature $T_{\epsilon}$ for the Narrow Line
Region (NLR) can be obtained from temperature sensitive diagnostic line ratios, e.g., [O III](4959+5007)/4363, while the electron number density $N_{\epsilon}$ from density sensitive line ratios, e.g., [O II] $3729 / 3726$ or [S II] 6716/6731. The earlier studies found $\log N_{\epsilon} \sim 2.0-2.8$ (Arribas \& Mediavilla 1993) \& 2.46 (Allen 1998); and $\mathrm{T}_{\epsilon}$ $\sim 18000 \mathrm{~K}$ (Arribas \& Mediavilla 1993) \& $14600 \mathrm{~K}$ (Evans et al. 1999). The integrated flux distribution of [S II]6716 \& [S II]6731 lines are shown in false color maps of Fig. 3(a) \& (b), while the line ratios and the resulting density maps are given in Fig. 3(c) \& (d). Here, we assumed $\mathrm{T}_{\epsilon}=15000 \mathrm{~K}$. The atomic constants are used from Keenan et al. (1996).

Fig. 3(a) \& (b) are from the integrated fluxes in the velocity range of $-180-+180 \mathrm{~km} \mathrm{~s}^{-1}$. To have a good $\mathrm{S} / \mathrm{N}$ density map, we ignored the zone of flux below $10 \%$ of the maximum. The $\mathrm{C}, \mathrm{NW}, \& \mathrm{~K}$ points and the reverse S-shaped boundary are marked in the density map. The higher density zone, i.e., $\log N_{\epsilon} \sim$ $3.1\left(1.3 \times 10^{3} \mathrm{~cm}^{-3}\right)$ occurs close to the $\mathrm{C}$ core. The 

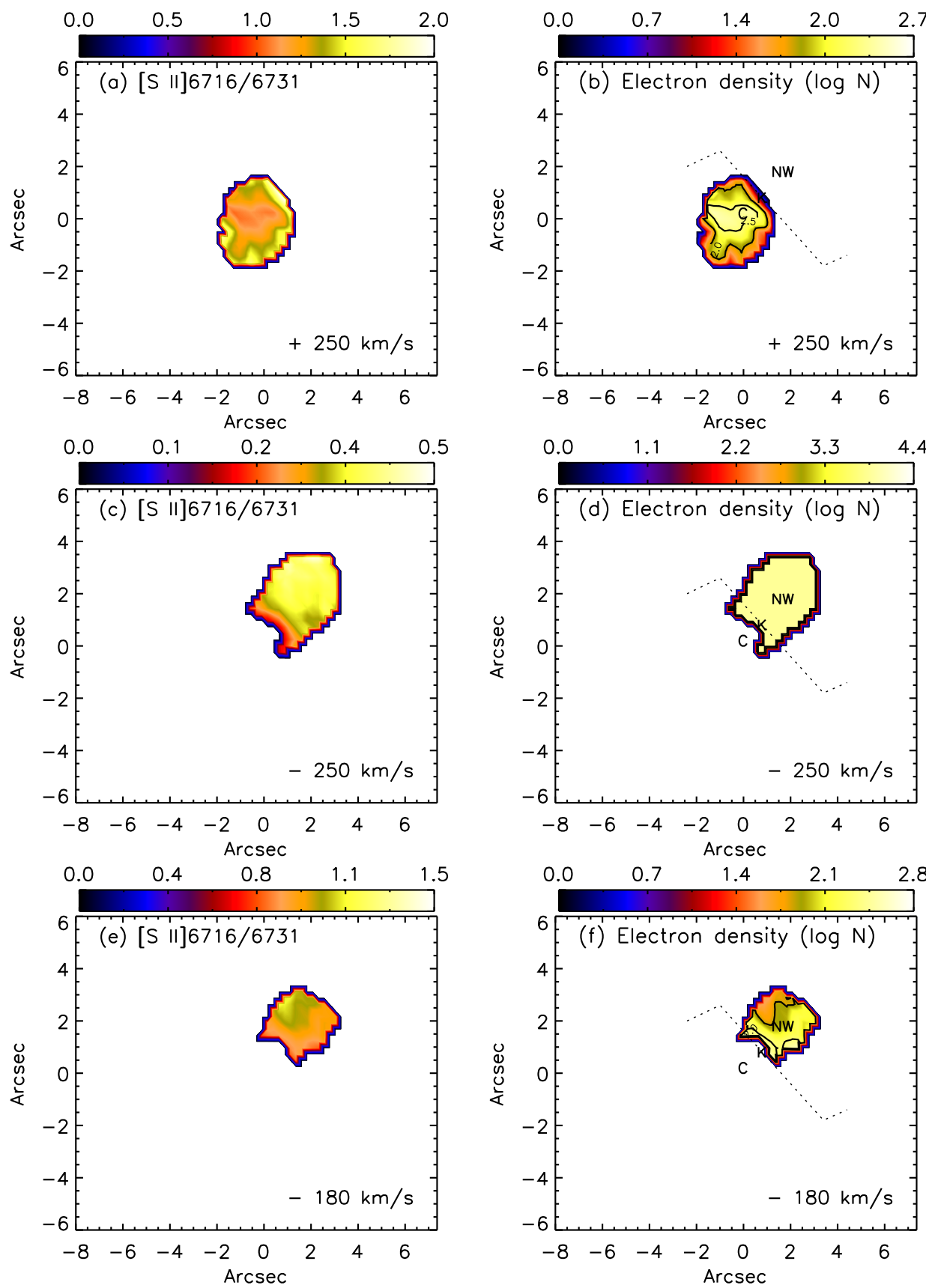

Fig. 4. - $[\mathrm{S}$ II $]$ ratios and electron density maps. (a) \& (b) at $\mathrm{V}_{\mathrm{r}}=+250 \mathrm{~km} \mathrm{~s}^{-1}$. (c) \& (d) $-250 \mathrm{~km} \mathrm{~s}^{-1}$. The integrated bandwidth is $1 \AA\left(\Delta \mathrm{V} \simeq 45 \mathrm{~km} \mathrm{~s}^{-1}\right)$. (e) \& (f) $-180 \mathrm{~km} \mathrm{~s}^{-1}$. The integrated bandwidth is $2 \AA\left(\Delta \mathrm{V} \simeq 90 \mathrm{~km} \mathrm{~s}^{-1}\right)$. Contour levels in (b) \& (f) are $2.0 \& 2.5$. 

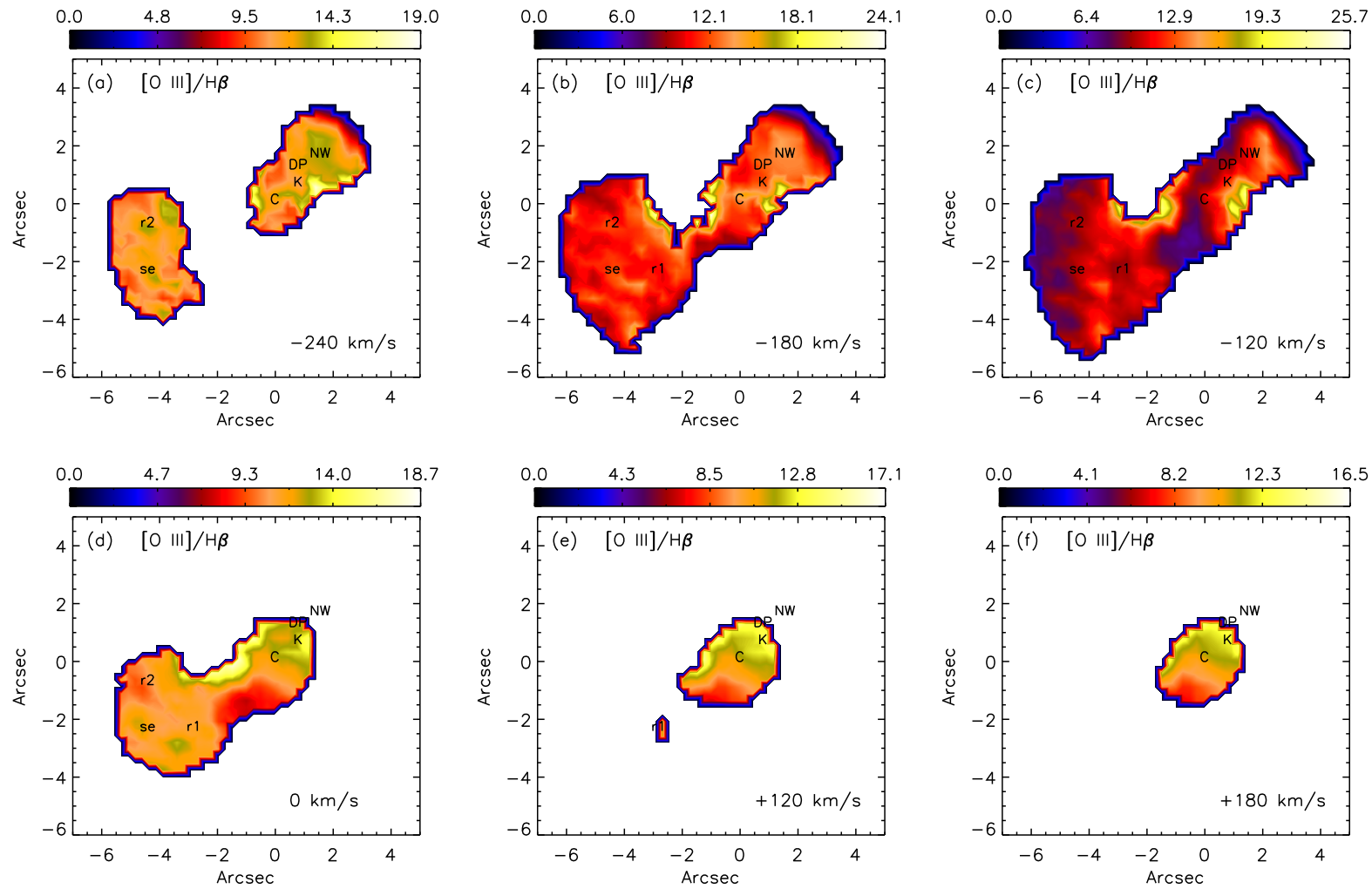

Fig. 5. - $[\mathrm{O}$ III $] 5007 / \mathrm{H} \beta$ ratios. The ratio greater than 3 indicates the region of AGN emission. See text.

resulting density map, however, is from a wide range of radial velocities, which represents the mixture of a wide range of spatial cross-sections. Hence, this value does not represent an actual spatial distribution of density within the circumnuclear zone.

According to Paper I, the emission of gas around the $\mathrm{C}$ core is stronger at $+250 \mathrm{~km} \mathrm{~s}^{-1}$, while that of the NW core is stronger at $-250 \mathrm{~km} \mathrm{~s}^{-1}$. Thus, we present other density maps in Fig. 4 at $\mathrm{V}_{\mathrm{r}}=-250 \&+250 \mathrm{~km}$ $\mathrm{s}^{-1}$, respectively. The density maps are obtained from the specific radial velocity slices within a narrow $1 \AA$ bandwidth $\left(\Delta \mathrm{V} \simeq 45 \mathrm{~km} \mathrm{~s}^{-1}\right)$. Strangely, the brighter $\mathrm{C}$ core's density, which is $\sim 500 \mathrm{~cm}^{-3}$, is lower than the relatively faint NW core value, $\sim 10^{4} \mathrm{~cm}^{-3}$. The derived value for the NW core zone might be in error, e.g., incomplete continuum elimination due to the weak [S II] fluxes. The other possibility is that the [S II] 6731 blue wing might be affected by the red wing of [S II] 6716 , resulting in a lower line ratio of $[\mathrm{S}$ II] 6716/6731 (and higher density). However, the $[\mathrm{O}$ III $] / \mathrm{H} \beta$ map at +180 $\mathrm{km} \mathrm{s}^{-1}$ in Fig. 5(f) does not show any strong AGN emission at the NW zone, which rules out the latter possibility.

We present $\mathrm{V}_{\mathrm{r}}=-180 \mathrm{~km} \mathrm{~s}^{-1}$ results in Fig. 4(e) \& (f). Here we adopted a bandwidth twice as wide as $2 \AA$. The result shows that the NW core density at $\mathrm{V}_{\mathrm{r}}$ $=-180 \mathrm{~km} \mathrm{~s}^{-1}$ is similar to the $\mathrm{C}$ core value at $\mathrm{V}_{\mathrm{r}}$
$=+250 \mathrm{~km} \mathrm{~s}^{-1}$. The higher density value at $\mathrm{V}_{\mathrm{r}}=$ $-250 \mathrm{~km} \mathrm{~s}^{-1}$ could be due to the ram pressure: the NW core might push out the galactic disk gas in front, and as a result the front zone might be compressed and highly heated. Our OASIS data, however, are not sophisticated enough to confirm this possibility.

The $[\mathrm{O}$ III] $] 5007 / \mathrm{H} \beta$ ratio of Seyfert 2 galaxies is known to be higher than 3 , while that of the $\mathrm{H}$ II region is lower (Osterbrock 1989). Fig. 5 shows the $[\mathrm{O}$ III $] 5007 / \mathrm{H} \beta$ ratios in false colors at different radial velocities. To have a good $\mathrm{S} / \mathrm{N}$ ratio map, we ignore lower flux zones, i.e., $20 \%$ or less of the maximum level. Note that the false color map does not indicate the stronger emission zone. These maps clearly show where the AGN emissions are dominant, i.e., $\mathrm{F}([\mathrm{O}$ III $] 5007) / \mathrm{F}(\mathrm{H} \beta)>3$. The NW core \& SE cone features are prominent in approaching radial velocity slices as in Fig. 5(a)-(c). The NW core disappears in Fig. 5(d)-(f). The size of the SE cone becomes smaller in Fig. 5(d) and disappears in Fig. 5(f).

\section{SEVERAL BLOBS AND CONES}

Numerous earlier studies pointed out the existence of a bi-conic structure, based on their integrated or large bandpass flux images. The HST images by Wilson et al. (1993) show that there is a well-defined cone struc- 

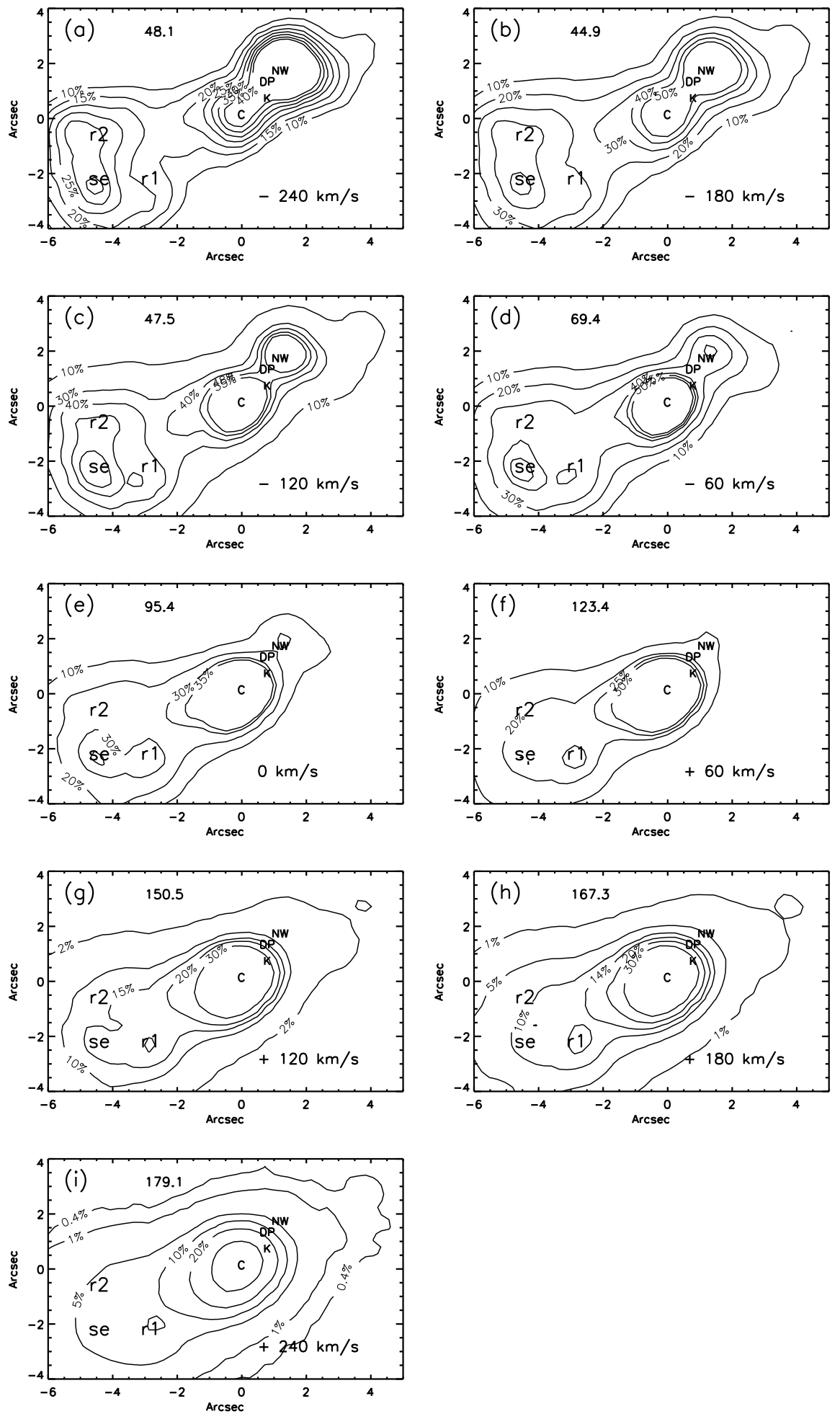

Fig. 6. - Several blobs in the $[\mathrm{O} \mathrm{III}]$ contour. (1) se blob: $\mathrm{V}_{\mathrm{m}}=-23 \mathrm{~km} \mathrm{~s}^{-1}$ at $(-4.4,-2.4) ;(2)$ ' $\mathrm{r} 1$ ' blob: $\mathrm{V}_{\mathrm{m}}=+50$ $\mathrm{km} \mathrm{s}^{-1}$ near $(-2.8,-2.4)$; (3) ' $\mathrm{r} 2$ ' blob: $\mathrm{V}_{\mathrm{m}}=12 \mathrm{~km} \mathrm{~s}^{-1}$ at $(-4.4,-0.8)$; (4) weak blob near (3.6, 2.8). The number at the top of each plot means the peak flux level in $10^{-19} \mathrm{~W} \mathrm{~m}^{-2} \operatorname{arcsec}^{-2}$. 
TABLE 1.

Measurements of 3 BLOBS IN [O III]5007

\begin{tabular}{llrll}
\hline \hline Position & $\mathrm{F}_{\mathrm{m}}^{\dagger}$ & $\mathrm{V}_{\mathrm{m}}\left(\mathrm{km} \mathrm{s}^{-1}\right)$ & $\mathrm{V}_{\mathrm{FWHM}}\left(\mathrm{km} \mathrm{s}^{-1}\right)$ & Fig. 6 (V $\mathrm{r})$ \\
\hline se $(-4.4,-2.4)$ & 33.4 & $-23.1 \pm 0.6$ & $429.0 \pm 1.4$ & (e) 0 \\
r1 (-2.8,-2.4) & 32.2 & $49.5 \pm 0.9$ & $421.4 \pm 2.2$ & (f) 60 \\
r2 $(-4.4,-0.8)$ & 26.7 & $11.5 \pm 0.8$ & $446.0 \pm 2.0$ & (e) 0 \\
\hline
\end{tabular}

$\dagger 10^{-19} \mathrm{~W} \mathrm{~m}^{-2} \operatorname{arcsec}^{-2}$

se: expanding blob in the cone

r1 \& r2: inflow blobs near the sky plane

ture expanding from the apex at K (see also Arribas \& Mediavilla 1993). Because of its close position to us, the large area gas in the expanding SE cone appears to be moving toward us (i.e., showing blue-shifted line profiles); whereas the out-flowing gas located farther away in a smaller NW cone will form red-shifted line profiles. The NW cone might be blocked mostly by the relatively thick galactic disk. As a result, the cone appears to be relatively fainter and smaller in scale.

Fig. 6 shows various [O III $]$ line-of-sight radial velocity contours. To search for minute structures in an extended conic area, we check various contour maps taken at different radial velocities. The radial velocity map morphologies are different in the blue- \& redshifted radial velocity slices. From the former blue contour images in Fig. 6(a)-(d), one can see a conspicuous box-shaped contour within the SE conic structure, e.g., $\sim 3^{\prime \prime} \times 4^{\prime \prime}$ at the $10 \%$ or $40 \%$ level $\left(4.8\right.$ or $19.0 \times 10^{-19} \mathrm{~W}$ $\mathrm{m}^{-2} \operatorname{arcsec}^{-2}$ ) in Fig. 6(c). Meanwhile, the red-shifted contour images in Fig. 6(f)-(i) are more complicated. The extended SE area must be carefully scrutinized to understand the kinematical behavior of the blobs. We identify several irregular blobs in the SE zone as 'se', 'r1', \& 'r2', based on Fig. 6.

The brightest 'se' blob at $(-4.4,-2.4)$ is 5 " away from the $\mathrm{C}$ position along the minor axis. The shape does not change much from one to another in various radial velocity slices. The size is about $1 .^{\prime \prime} 2 \times 1$.' $^{\prime \prime}$ at the $40 \%$ level in Fig. 6(d). Table 1 presents the FWHM of line profiles for 'se' and other 2 blobs. The FWHM of the 'se' blob line profile is very large; $\mathrm{V}_{\mathrm{FWHM}}=429 \mathrm{~km}$ $\mathrm{s}^{-1}$. The 'se' blob appears in all of the radial velocity slices in Fig. 6(a)-(i). The 'se' blob which has a peak radial velocity of $\mathrm{V}_{\mathrm{m}}=-23 \mathrm{~km} \mathrm{~s}^{-1}$, mostly approaches us. It behaves similar to the large extended SE cone itself.

Fig. 7 schematically shows the position and rough size of three blobs in a geometric model of NGC 5728 circumnuclear zone. Their actual size and kinematical structure would be more complicated. Paper I concluded that the $\mathrm{C} \& \mathrm{NW}$ cores are a gravitationally bound system; the $\mathrm{C}$ core is moving away from us, while the NW core is approaching us. The $r=5 !^{\prime \prime} 4$ inner NW ring and the galactic plane are nearly par- allel $\left(i \sim 50^{\circ}\right.$ relative to the observer). The C \& NW cores, the NW 5!" 4 ring, the two cones, and the galactic disk are shown in this geometric model, based on the Paper I's result. The 'se' blob must be located within the opening angle of the SE cone as in Fig. 7, which might expand along the polar axis.

The second brightest blob at $(-2.8,-2.4)$, marked as ' $r 1$ ' in figures, shows both receding \& approaching radial velocity components. The maximum flux occurs at radial velocity, $\mathrm{V}_{\mathrm{m}}=+50 \mathrm{~km} \mathrm{~s}^{-1}$ (see Fig. $6(\mathrm{f})$ ). This ' $\mathrm{r} 1$ ' blob is weak relative to the 'se' blob, but strong enough to define its own boundary. The overall blob size is less than $1^{\prime \prime}$ at a distance of $33^{\prime \prime} 7$ from $\mathrm{C}$ along $\mathrm{PA} \sim 130^{\circ}$ (see Fig. 6(c)-(i)). Note that the maximum flux position of the blob is shifting from $(-3.5,-2.5)$ at $\mathrm{V}_{\mathrm{r}}=-120 \mathrm{~km} \mathrm{~s}^{-1}$ contour map into a nearby $(-2.5,-2.0)$ at $\mathrm{V}_{\mathrm{r}}=+240 \mathrm{~km} \mathrm{~s}^{-1}$ contour map. This is likely to be due to the lengthy streaming (or stretched) flow of the gas cloud. Apparently, the 'r1' blob could be a filamentary-like lengthy cloud depicted in Fig. 7. The whole structure may be flowing into the center which is in the $\mathrm{PA}=-60^{\circ}$ direction. The close part of this lengthy gas cloud, however, appears to be receding $(+)$ from us, while the distant part appears to be approaching $(-)$. See $(-) \&(+)$ signs in Fig. 7.

The ' $\mathrm{r} 1$ ' blob might be an inflow into $\mathrm{C}$ (or $\mathrm{K}$ ) and as a result we are able to see its maximum flux positional change in radial velocity slice maps. In this case, the front portion (or the closer side to the observer) of the elongated gas blob is responsible for the red-shifted contour image in Fig. 6(i), while the rear portion of the blob gas is responsible for the blue-shifted contour, i.e., the distant part of the 'r1' blob in Fig. 6(c). The inset in Fig. 7 shows an opposite outflow interpretation. With the outflow model, one can explain similarly the positional shift of the ' $r 1$ ' blob seen in Fig. 6: the left side of the blob in model diagram is responsible for the red velocity slice contour, though. If this is the case, one might interpret ' $\mathrm{r} 1$ ' as a remnant or broken part of a (hot) bubble. The huge range of velocity in 'r1' might be related to the kinematics of an expanding large bubble. However, any further speculation is beyond the current study limit. 


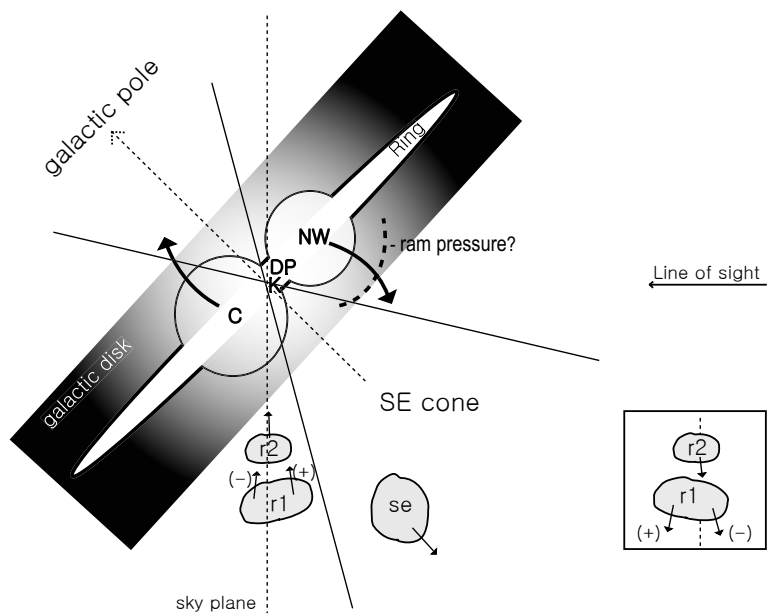

Fig. 7. -3 blobs in a circumnuclear geometric model. The schematic model for NGC 5728 circumnuclear zone is from Paper I. The observer is on the right side in this diagram. The vertical line is named as 'sky plane', since it is parallel to the sky plane.

There is a third blob at $(-4.4,-0.8)$, named ' $\mathrm{r} 2$ ', which is at a distance of $4 .^{\prime \prime} 5$ from $\mathrm{C}$ along $\mathrm{PA} \sim 100^{\circ}$. Its maximum flux occurs at $\mathrm{V}_{\mathrm{m}}=+12 \mathrm{~km} \mathrm{~s}^{-1}$, which is close to the system velocity. The shape is not clear in contour maps, but its presence is obvious in the false color enhanced images in Fig. 1(a) \& (b). Its characteristic is similar to that of ' $\mathrm{r} 1$ ', but its location is closer to C. In the integrated contour map, the ' $\mathrm{r} 2$ ' blob appears to belong to the 'se' blob or a part of a relatively large 'se' blob family, but the line-of-sight radial velocities largely differ. In Fig. 7, we mark the 'r2' blob position close to the sky plane, which is far from the SE cone axis. The ' $r 2$ ' blob could be another cloud which also approaches the center due to the strong gravitational influence of the two rotating core systems. Both ' $\mathrm{r} 1$ ' \& 'r2' appear to be close to the sky plane and slightly receding from the observer, but the 'se' blob is likely to be part of the SE cone.

There appears to be another blob at $(3.6,2.8)$ in the NW conic zone. See the $+120-+240 \mathrm{~km} \mathrm{~s}^{-1}$ images in Fig. 6(g)-(i). This one is at a distance of $4 .^{\prime \prime} 6$ from $\mathrm{C}$, which is located inside the $\mathrm{H} \alpha \mathrm{NW}$ ring. This blob may be part of a jet-like structure protruding toward the NW direction, seen in Fabry-Perot $\mathrm{H} \alpha$ contour and radio maps by Schommer et al. (1988).

The approaching $\mathrm{V}_{\mathrm{r}}=-120 \mathrm{~km} \mathrm{~s}^{-1}$ velocity slice or most other contours in Fig. 6 clearly indicate the presence of a strong SE cone (10\% level), while the corresponding contour at $\mathrm{V}_{\mathrm{r}}=+120 \mathrm{~km} \mathrm{~s}^{-1}$ in Fig. 6 (g) barely shows the NW cone (2\% level). The opening angle of the cone is quite large, i.e., $>50^{\circ}$ in the SE direction (Wilson et al., 1993), but it is undefinable in the NE direction.

Some part of the 'se', and 'r1' \& 'r2' blobs are still seen in $\mathrm{V}_{\mathrm{r}}=0 \mathrm{~km} \mathrm{~s}^{-1}$ (Fig. $5(\mathrm{~d})$ ), but only the ' $\mathrm{r} 1$ ' blob remains in $\mathrm{V}_{\mathrm{r}}=+120 \mathrm{~km} \mathrm{~s}^{-1}$ (Fig. 5(e)). The $\mathrm{C}$ core is seen in the highest speed receding velocity map in Fig. $5(\mathrm{f})$. The $[\mathrm{O}$ III $] 5007 / \mathrm{H} \beta$ ratio map tends to support our earlier interpretation on the flowing direction of the 3 blobs.

As seen in Fig. 5, the gas around two cores \& K zone is likely to be excited by the AGN hot source(s). The SE cone and blobs are also perhaps excited by the same AGN source. The gas at a relatively large scale might flow into the kinematical center, while the gas in the polar cone zone might expand as mentioned earlier. However, we also must not forget the rotating behavior of two cores: the receding $\mathrm{C}$ core and the approaching NW core. There appears to be a compressed hot shell affected by ram pressure in front of the rotating NW core. All of the above mentioned kinematical structures must be incorporated in the circumnuclear structure of NGC 5728.

The appearance of the $\mathrm{C}$ core in Fig. 6(d)-(h) displays an elongated sphere shape. The largest diameter of the ellipsoidal core is along $\mathrm{PA} \simeq-10^{\circ}$, which is about the middle of the major and minor axes of the disk. The core's specific directional elongation may be caused by a tidal force or rotation of the innermost gas within the circumnuclear zone. The distorted structure of the disk had been noted in other studies, e.g., Rubin (1980). The elongation might be related to the gas along the galactic plane which swirls into the center from a larger distance. The blobs ' $\mathrm{r} 1$ ' \& ' $\mathrm{r} 2$ ' appear to be approaching the center, while the 'se' blob is part of the expanding SE cone along the polar axis. Although we temporarily conclude that ' $\mathrm{r} 1$ ' \& ' $\mathrm{r} 2$ ' are outflows to harmonize with the kinematics for the two cores and the inner $3^{\prime \prime}$ radius disk discussed in Paper I, this still remains speculative unless there is another independent way to confirm this.

\section{CONCLUSIONS}

We derived the physical condition of the nucleus zone of NGC 5728 using the IUE and OASIS data; we identified several blobs and investigated their flow activities. Careful examination of various radial velocity contours and OASIS [S II], [O III], \& $\mathrm{H} \beta$ spectral data imply that the circumnuclear zone consists of numerous complex substructures in different orientations, involving small blobs or filamentaries.

The [S II] diagnostics imply that the overall gas electron density around both the $\mathrm{C} \& \mathrm{NW}$ cores is about $500 \mathrm{~cm}^{-3}$. However, the $[\mathrm{S} \mathrm{II}]$ diagnostics at $\mathrm{V}_{\mathrm{r}}=-250$ $\mathrm{km} \mathrm{s}^{-1}$ implies that there is a higher electron density shell in front of the NW core's rotating direction. It could be a ram pressure zone, implying interaction of the rotating NW core with the galactic gaseous material in front. The jet-like structure protruding toward the NW direction (Schommer et al. 1988) might be related to this kinematical structure.

Paper I showed that the inner $r=3^{\prime \prime}$ disk $\left(i \sim 55^{\circ} .5\right.$, 
$\mathrm{PA}(\mathrm{a})=123^{\circ}$ ) gas around the two main cores is flowing into the center, while the $r=5 .^{\prime \prime} 4 \mathrm{NW}$ ring $\left(i \sim 50^{\circ}\right.$, $\mathrm{PA}(\mathrm{a})=20^{\circ}$; nearly parallel to the galactic plane) is expanding. Based on our analysis of the [O III] radial velocity data, we deduced the loci of 3 blobs on Paper I's model geometry. We found that the ' $r 1$ ' \& ' $r 2$ ' blobs outside the SE cone are inflowing into the center, while the 'se' blob within the SE cone is flowing out.

Is there any possibility that ' $\mathrm{r} 1$ ' \& ' $\mathrm{r} 2$ ' and other inner most gases are outflowing instead of inflowing? Such a possibility still exists (see the inset in Fig. 7), although various kinematical properties for other important structures found in Paper I seem to deny the possibility of such outflow activity. All the circumnuclear structures may have been formed due to the gravitational influence of the center of mass of the two main cores. The ' $r 1$ ' \& ' $r 2$ ' blobs could be transitory structures generated by the gravitational force of the two core system. To further investigate the transition from a relatively large scale slow inflow to a sub-arcsec scale fast inflow, much more sophisticated observations, using SAURON or VLBI, would be desirable.

S. H. acknowledges Korea Astronomy and Space Science Institute (KASI) for the 20090415 research grant to Chungbuk National University. We thank Eugenia H. of Ohio State University for her proof-reading and the anonymous referee for his/her careful review.

\section{REFERENCES}

Allen, M. G., 1998, PhD thesis, The Australian National University

Arribas, S. \& Mediavilla, E., 1993, The circumstellar region of NGC 5728, ApJ, 410, 552

Evans, I., Koratkar, A., Allen, M., Dopita, M., \& Tsvetanov, Z., 1999, The Excitation Mechanism of Emission-Line Regions in Seyfert Galaxies, ApJ, 521,531

Feibelman, W. A. \& Aller, L. H., 1987, The (C III lambda 1909/Si III lambda 1892) ratio as a diagnostic for planetary nebulae and symbiotic stars, ApJ, 319,407

Hyung, S., Kim, H., Lee, W. B., Lee, S.-J., Ryu, D., \& Lee, H.-W., 2000, IUE Spectra of the Seyfert 1 Galaxies Mrk 335 and NGC 4051, JKAS, 33, 81

Keenan, F. P., Aller, L. H., Bell, K. L., Hyung, S., McKenna, F. C., \& Ramsbottom, C. A., 1996, Auroral and nebular emission lines of [SII] in the optical spectra of planetary nebulae, MNRAS, 281, 1073

Mediavilla, E., \& Arribas, S., 1995, Two-dimensional spectroscopy of the Seyfert galaxies NGC 5728 and 4151 in the [OIII] lambdalambda4959, 5007 and $\mathrm{H}$ spectral ranges, MNRAS, 276, 579

Osterbrock, D. E., 1989, Astrophysics of Gaseous Nebulae and Active Galatic Nuclei, University Science Books, Sausalito, California
Pécontal, E., Adam, G., Bacon, R., Courtes, G. Georgelin, Y., \& Monnet, G., 1990, Observation of the central region of NGC 5728 with the integral field spectrograph TIGER, A\&A, 232, 331

Rubin, V. C., 1980, Velocities and mass distribution in the barred spiral NGC 5728, ApJ, 238, 808

Schommer, R. A., Caldwell, N., Wilson, A. S., Baldwin, J. A., Phillips, M. M., Williams, T. B., \& Turtle, A. J., 1988, Ionized gas and radio emission in the barred Seyfert galaxy NGC 5728, ApJ, 324, 154

Son. D.-H., Hyung, S., Ferruit, P., Pécontal, E., \& Lee, W.-B., 2009, The kinematics of the Seyfert 2 galaxy NGC5728 circumnuclear region, MNRAS, 395, 692 (Paper I)

Wilson, A. S., Braatz, J. A., Heckman, T. M., Krolik, J. H., \& Miley, G. K., 1993, The Ionization Cones in the Seyfert Galaxy NGC 5728, ApJ, 419, L61 